\title{
Practical Considerations for Community-Based Health Promotion Events during the COVID-19 Pandemic
}

Sarah E. Vordenberg, PharmD, MPH ${ }^{1,2}$; Heidi L. Diez, PharmD ${ }^{1,3}$; Caitlin Ferguson, MA ${ }^{1 ;}$ Paul C. Walker, PharmD ${ }^{1,4}$; Jolene R. Bostwick, PharmD ${ }^{1,4}$; Kristin C. Klein, PharmD ${ }^{1,4}$

${ }^{1}$ University of Michigan College of Pharmacy; ${ }^{2}$ University of Michigan Center for Bioethics and Social Sciences in Medicine;

${ }^{3}$ Michigan Medicine Pharmacy Innovations and Partnerships; ${ }^{4}$ Michigan Medicine Department of Pharmacy

\begin{abstract}
Community-based health promotion events provide student pharmacists the opportunity to give back to the local community while simultaneously applying the knowledge and skills they are learning in the classroom (Accreditation Council for Pharmacy Education Standards 3, 4, and 12). In turn, community members receive benefits, such as receiving a vaccination and learning their blood pressure, as well as strategies to manage their health conditions. Traditionally, both individual community members and student pharmacists receive benefit. As a result of the coronavirus disease 2019 (COVID-19) pandemic, it is critical to consider the impact of public health via the local community when choosing to hold or suspend these activities. It is necessary to consider whether the benefits to individual community members who choose to participate (e.g., older adult with type 2 diabetes or underserved adults with limited access to the influenza vaccine) outweigh the risks to the public due to the pandemic. If there is sufficient benefit, there are practical considerations related to regulations, recruitment of community members, involvement of students and preceptors, location, supplies, delivery of patient care services, and activities after the event.
\end{abstract}

Keywords: community engagement, COVID-19, co-curricular, pharmacy student, public health

\section{Introduction}

Student pharmacists routinely participate in community health events (referred to as "health events") providing services such as education about the signs and symptoms of stroke; blood pressure, blood glucose, and cholesterol screenings; immunizations delivery; and medication counseling. These events provide benefits to both the community and the students. Studies have demonstrated improvements in community participants knowledge of stroke symptoms, diabetes, and hypertension. ${ }^{1,2}$ One study demonstrated $78 \%$ of participants in a health event with abnormal values planned to follow up with their healthcare providers. ${ }^{1}$

The Accreditation Council for Pharmacy Education (ACPE) Standards 2016 require pharmacy students to complete 300 hours of introductory pharmacy practice experience (IPPE) hours, half of which should be distributed evenly between community and health system care (standard 12$).{ }^{3}$ Standards 2016 also endorse the use of co-curricular activities, including health events, to fulfill standards 3 (approach to practice and care), 4 (personal and professional development), and 12 (preadvanced pharmacy practice experiences curriculum). ${ }^{3}$ Students benefit from participation in health events by improving their confidence in caring for members of the targeted community, completion of required IPPE hours, and participation in co-curricular events to foster their professional development. ${ }^{4}$

Corresponding author: Sarah E. Vordenberg, PharmD, MPH University of Michigan College of Pharmacy

P: 734-763-6691; Email: skelling@med.umich.edu
The coronavirus disease 2019 (COVID-19) pandemic has impacted the framework for planning and conducting community-based health promotion events. Institutions need to balance students' needs for IPPE hours and professional development with the risk of exposure to COVID-19 to students, preceptors, and community members. Below are practical factors that institutions may consider when determining whether to hold a health event (Tables 1 and 2).

\section{Considerations for health events}

Outlining goals and exploring options

When beginning planning for a health event, it is important to consider the event goals for students, community members, and public health. This will help to determine if an in-person event is necessary (e.g., administering an influenza vaccine) or whether using an alternative, such as videoconferencing, may be a viable option (e.g., providing education about tobacco cessation). ${ }^{5}$ For populations with limited access to technology or low technology literacy, a hybrid model in which a small number of students take computers with Internet access to the event location while other students work remotely to provide the service may be possible.

\section{Legal considerations}

If in-person events are being considered, it is imperative to frequently monitor local conditions, laws, regulations, and guidelines from university, city, state, and federal authorities as the pandemic is dynamic and changes may quickly occur. ${ }^{6}$ If a pharmacy program is partnering with an external organization, all parties should discuss ahead of time shared decision-making related to when to cancel events and how to notify community members. ${ }^{7}$ Consider talking with the local public health 
department to determine next steps, if a community member who may have COVID-19 comes to the event and establish an agreed upon plan for contact tracing. Pharmacy programs should consider reaching out to their legal department to determine if general consent forms for patient or student participation should be revised to address risks related to COVID-19.

\section{Community members}

It is important to consider whether the targeted patient population will receive sufficient benefit from the health event to outweigh the risks of potential exposure to COVID-19. For example, people at increased risk for severe illness from COVID19 include those with chronic kidney disease, chronic obstructive pulmonary disease, heart failure, obesity, and type 2 diabetes mellitus. ${ }^{8}$ In addition, risk increases with advancing age, and more than $80 \%$ of deaths from COVID-19 in the United States have been in people 65 years and older. ${ }^{9}$ While older people and those with chronic conditions may receive medical or social value from participating in health events, they are also at higher risk for morbidity and mortality from COVID-19, particularly if a large number of people are present or they are onsite for a long time. Furthermore, the purpose of some events is to screen patients to identify if they may have a condition (e.g., blood glucose screening to identify a person with diabetes). Individuals with an abnormal result may not have realized they were at higher risk for COVID-19, thus, may have additional questions that will need to be addressed.

When determining whether to hold a health event, it may be helpful to outline what benefit patient populations may receive from participating in the health event, alternatives to receive the same service, and the anticipated risks due to COVID-19. For example, there may be sufficient benefit to hold an influenza immunization clinic for migrant workers without access to routine healthcare services. In contrast, the risks of COVID-19 exposure may outweigh the benefits when holding a blood glucose screening clinic for older adults in a high-income community with strong access to the health care system. The risks and benefits trade-off may vary based on the type of health service provided, patient population, and level of community transmission of COVID-19. The World Health Organization has a comprehensive risk assessment tool that may be useful. ${ }^{10}$

\section{Number of participants}

Generally, one factor that is used to measure the success of the event is the number of participants. However, the higher the number of participants, the higher the risk of COVID-19 transmission. Therefore, it may be reasonable to intentionally limit the size of events during the pandemic. One strategy to help manage the number of people throughout the event is to ask that community members sign up for appointments. This could help to support appropriate spacing between patients, time for sanitation procedures between each patient, and limit patient congregation prior to the event. Furthermore, having a list of participants with telephone numbers may help with contract tracing, if necessary. ${ }^{11}$ The process for signing up should comply with the Health Insurance Portability and Accountability Act (HIPAA). It is also important to outline a strategy for managing people who have not signed up in advance, such as allowing them to participate, only if there is an open appointment slot. Qualitative feedback from community members may help to demonstrate the value of the event and identify opportunities for improvement in the future.

\section{Students and preceptors}

Students and preceptors should consider their personal health status, and those of their close contacts, when considering whether to engage in health events. Institutions should consider whether to make these types of health events optional or required, and if the later, have a policy in place for individuals to defer their participation, if needed. All students and preceptors should receive instructions about appropriate donning and doffing of personal protective equipment prior to the event. ${ }^{12,13}$ Students and preceptors should be instructed not to attend the event if they feel ill or have had a positive COVID-19 test within the time period designated by the pharmacy program. Institutional policies, such as those related to temperature screening, symptom screening, and recording of these results should be followed, even if the event is not held on campus. For example, at our institution, students and preceptors must complete a screening on our institution's cellphone application or website prior to participating in health events as well as an additional student registration form. A list of all students and preceptors should be obtained at the beginning of the event. Student shifts should be staggered so that the number of people on site (e.g., starting and finishing their shifts) can be limited.

\section{Location}

Prior to COVID-19, many health events took place indoors. While it may be possible to offer outdoor events, both locations have unique challenges and considerations. For indoor events, it is important to consider room capacity, the ventilation system, and movement through the clinic so that there is one entrance and one exit. ${ }^{14}$ Guidelines regarding the number of square feet per person will determine how many people can be in one room at a given time. For example, if an institution requires spacing of 36 square feet per person for an event in which all participants are seated, a medium-sized room of $12 \times 12$ feet can only hold four people. This would likely include a preceptor, a student, and up to two community members (e.g., one receiving the patient care service and a second waiting their turn). However, if people are moving throughout this event, the room would only be appropriate for 1 person if the institution required 144 square feet per person. These space constraints significantly limit the number of community members that can be seen during a health event, which may lead to longer events or the use of multiple rooms simultaneously to serve the same number of community members. Longer shifts could also result in the need for more 
student volunteers and preceptors. If more rooms are used, additional preceptors may be needed to observe students. If volunteer shifts are long, a break may be needed for a meal and, therefore, a space for a break may need to be designated.

Outdoor events are subject to weather conditions, and it may be necessary to purchase or lease equipment such as tents, chairs, and tables. A standard canopy tent is $10 \times 10$ feet, which means that only one student and one community member can be in the tent, thus posing a precepting issue. If the weather is hot or cold, equipment such as glucometers and test-strips can malfunction. Extreme temperatures can also negatively impact the volunteers who may be present for multiple hours, as well as community members (e.g., older adults). Therefore extra steps, such as distribution of sealed water bottles in hot weather or access to outdoor heaters in cold weather, will need to be taken to ensure safety. Restrooms with running water should be accessible for students, preceptors, and community members, although this presents physical distancing and cleaning challenges.

\section{Supplies}

Health event kits with essential supplies should be provided onsite. The kit may include physical distancing signs, extra masks, face shields and/or protective eyewear for students and preceptors, cleaning supplies, hand-sanitizers for each station, and sanitized screening equipment. If the sponsoring organization is providing these supplies, cost and waste should be considered.

\section{Delivery of patient care services}

Upon arrival, community members should immediately go to a station that includes a verbal COVID-19 symptom screen and possible temperature check with a non-contact infrared thermometer. ${ }^{15}$ Individuals who may have COVID-19 should be provided information about when and where to seek care and be asked to leave the event. A mask should be provided, if any person arrives without one, and all participants should be instructed that it must be worn over the mouth and nose while on the premise. ${ }^{16} \mathrm{~A}$ record should be maintained of each person who attends the event, including their contact information in case contact tracing is necessary.

After this station, community members can be provided with a sticker indicating their negative screening status. Community members should stand or sit in the waiting area with chairs placed six feet apart or the floor marked with tape at 6-foot intervals. Volunteers may need to provide verbal reminders to community members about the importance of physical distancing. Chairs should be periodically cleaned and hand sanitizer should be available.

Once seated, community members should be instructed to sanitize their hands. Students conducting the service may be required to wear gloves for the duration of the interaction, as well as masks and protective eyewear/face shield. Neither the student nor community member should lower their masks, even if one would typically do so, such as while demonstrating how to use an inhaler. Once the service is completed, students should sanitize the equipment, tables, and chairs, and change gloves before providing care to the next community member. ${ }^{17}$

\section{Post-event}

After the event, tables and chairs should be cleaned. Reusable equipment should be sanitized, labeled, and repacked. An inventory should be maintained of all consumable supplies, such as medical masks and face shields so that re-ordering can happen in a timely manner, given the potential delays in delivery that may occur due to high demand. The list of student volunteers, preceptors, and community members should be maintained in a secure location. Consider including detailed documentation of individual interactions, if people are within close contact (e.g., less than 6 feet), particularly for extended periods of time (e.g., longer than 15 minutes), All documents must be stored in a HIPAA-compliant location. Finally, programs should follow guidance from their institution and the local public health department, if there are concerns about potential COVID-19 exposure based on testing that occurs after the health event.

\section{Conclusion}

Community-based health promotion events provide student pharmacists the opportunity to give back to the local community, while simultaneously applying the knowledge and skills they are learning in the classroom setting. In turn, community members receive benefits, such as receiving a vaccine, learning their blood pressure, and learning strategies to manage their health conditions. Traditionally, both individual community members and student pharmacists receive benefit. As a result of the COVID-19 pandemic, it is critical to consider the impact on public health via the local community when choosing to hold or suspend these activities. If an event is able to be held, there are considerations related to regulations, recruitment of community members, involvement of students and preceptors, location, supplies, delivery of patient care services, and activities after the event.

\author{
Funding source: None \\ Financial disclosures and conflicts of interest: None \\ Treatment of human subjects: Not applicable
}




\section{References}

1. Hess KM, Gabrielian C, Schwartzman E, Law AV. The impact of student pharmacists at health fair events. Am J Pharm Educ. 2012;76(8):article 149.

2. Vo LP, Souksavong JH, Tran A, Chang J, Lor KB. Impact of Act FAST stroke campaign delivered by student pharmacists on the primary prevention of stroke. J Am Pharm Assoc. 2017;57:326-32.

3. Accreditation Council for Pharmacy Education. Accreditation standards and guidelines for the professional program in pharmacy leading to the Doctor of Pharmacy degree. Chicago, IL: Accreditation Council for Pharmacy Education, 2015. Retrieved from https://www.acpeaccredit.org/pdf/Standards2016FINAL.pdf Accessed 16 August 2020.

4. Janzen K, Kormelink LN, Saum L, Nisly SA. Geriatric and student perceptions following student-led educational sessions. Innov Pharm. 2018;9(1):article 1.

5. COVID-19: Telehealth and virtual care best practices. American Hospital Association. Retrieved from https://www.aha.org/system/files/media/file/2020/04/CO VID-19-Telehealth-Best-Practices final.pdf. Accessed 17 August 2020.

6. Information for healthcare professionals about coronavirus (COVID-19). Centers for Disease Control and Prevention. Retrieved from https://www.cdc.gov/coronavirus/2019nCoV/hcp/index.html. Accessed 18 August 2020.

7. Section 9. Making decisions. Community Tool Box. Retrieved from https://ctb.ku.edu/en/table-ofcontents/leadership/leadership-functions/makedecisions/main. Accessed 17 August 2020.

8. People with certain medical conditions. Centers for Disease Control and Prevention. Retrieved from https://www.cdc.gov/coronavirus/2019-ncov/need-extraprecautions/people-with-medical-conditions.html. Accessed 10 August 2020.

9. Older adults. Centers for Disease Control and Prevention. Retrieved from https://www.cdc.gov/coronavirus/2019ncov/need-extra-precautions/older-adults.html. Accessed 10 August 2020.
10. WHO mass gathering COVID-19 risk assessment tool: Generic events. World Health Organization. Retrieved from https://www.who.int/publications/i/item/10665-333185. Accessed 17 August 2020.

11. Case investigation and contact tracing: Part of a multipronged approach to fight the COVID-19 pandemic. Centers for Disease Control and Prevention. Retrieved from https://www.cdc.gov/coronavirus/2019ncov/php/principles-contact-tracing.html. Accessed 17 August 2020.

12. How to safely put on personal protective equipment (PPE). Centers for Disease Control and Prevention. Retrieved from https://www.youtube.com/watch?v=H4jQUBAlBrl. Accessed 17 August 2020.

13. How to safely take off personal protective equipment (PPE). Centers for Disease Control and Prevention. Retrieved from https://www.youtube.com/watch?v=PQxOc13DxvQ. Accessed 17 August 2020.

14. COVID-19 employer information for office buildings. Centers for Disease Control and Prevention. Retrieved from https://www.cdc.gov/coronavirus/2019ncov/community/office-buildings.html. Accessed 17 August 2020.

15. Symptoms of coronavirus. Centers for Disease Control and Prevention. Retrieved from https://www.cdc.gov/coronavirus/2019-ncov/symptomstesting/symptoms.html. Accessed 17 August 2020.

16. How to wear masks. Centers for Disease Control and Prevention. Retrieved from https://www.cdc.gov/coronavirus/2019-ncov/preventgetting-sick/how-to-wear-cloth-face-coverings.html. Accessed 17 August 2020.

17. Cleaning and disinfecting your facility. Centers for Disease Control and Prevention. Retrieved from https://www.cdc.gov/coronavirus/2019ncov/community/disinfecting-building-facility.html. Accessed 17 August 2020. 
Table 1. Factors to weigh when deciding whether to hold a community health event during the COVID-19 pandemic

Outlining goals and exploring options

- Establish the goals for students, community members, and public health

- Determine whether the goal can be met using alternative approaches, such as videoconferencing

Legal considerations

- Monitor local conditions related to COVID-19 transmission

- Monitor laws, regulations, and guidelines from the university, city, state, and federal authorities

- Establish a plan for shared-decision making with the community partner

- Talk with the public health department to determine appropriate steps if a person with potential COVID-19 presents to a community event

\section{Community members}

- Determine if the targeted patient population is at high risk for severe illness from COVID-19

- Outline and weight potential benefits, alternatives, and risks for community members participating in the event

- Consider using the World Health Organization comprehensive risk assessment tool for mass gatherings 
Table 2. Planning and implementing a community health event during the COVID-19 pandemic

Number of participants

- Consider requiring community members to sign up for events to support appropriate spacing, time for cleaning, and limiting congregation

Students and preceptors

- Encourage students and preceptors to consider personal health status, and those of close contacts, when considering whether to participate

- Institutions who require participation should develop a policy for individuals to defer their participation, if needed

- Identify which personal protective equipment is needed based on the role of each student and preceptor during the event

- Provide instruction to students and preceptors regarding donning and doffing of personal protective equipment

- $\quad$ Provide instructions about not participating, if the student or preceptor feels ill or had a positive COVID-19 test

- Obtain a list of all students and preceptor who attended the event and the times they arrived and left

Location

- Identify the room capacity based on square footage and amount of movement of people, concerns regarding the ventilation system, and potential flow of the clinic

- $\quad$ Establish the appropriate number of preceptors, keeping in mind the physical distancing may make it difficult to precept multiple students simultaneously

- Account for more space and/or a longer event, if high community turn out is expected

- Create a dedicated break area in which people can safely eat without a mask

Special considerations for outdoor events

- Obtain the necessary equipment such as tents, chairs, and tables

- Create a plan for inclement weather (e.g., rain, extreme heat or cold)

- Distribute sealed water bottles to community members, students, and preceptors, if it is hot

- Ensure access to restrooms with running water that meet cleaning and physical distancing requirements

Supplies

- $\quad$ Bring physical distancing signs, extra masks, face shields and/or protective eyewear, cleaning supplies, hand sanitizer for each station, and sanitized screening equipment

\section{Delivery of patient care}

- $\quad$ Direct community members to the screening station in which a trained student or preceptor can conduct a verbal screening for COVID-19 symptoms and possible temperature check with a non-contact infrared thermometer

- Instruct community members to wear a mask, which may need to be provided by the pharmacy program, throughout the event

- Collect contact information for each community member who attends the event (regardless of whether or not they participate in the health service)

- Community members should stand or sit in the waiting area with chairs or markings with tape placed 6 feet apart

- Community members should sanitize their hands at the beginning of the health event

- After the service is complete, students should sanitize the equipment, tables, and chairs, and change gloves

\section{Post-event}

- $\quad$ Clean tables, chairs, and reusable equipment

- Inventory and re-order consumable supplies in a timely manner

- Maintain a list of students, preceptors, and community members in a secure location 
Box 1. Example of decision making related to community health event during COVID-19 pandemic

\section{Outlining goals and exploring options}

Our College of Pharmacy has a partnership with an apartment building for low-income adults in our local community. We typically host an onsite monthly blood pressure and blood glucose screening and education event for apartment residents. Our long-term goal is to provide a holistic set of community-anchored clinical services and health promotion interventions for prevention and monitoring of chronic conditions, delivered by interprofessional student teams. The students who typically participate in this experience are from the pharmacy, undergraduate nursing, and graduate social work programs at our institution.

At the beginning of the pandemic, the College of Pharmacy cancelled all in-person health events with a few exceptions (e.g. flu clinic and events tied to a medical facility). Our team explored implementing virtual visits related to hypertension management. However, we identified a number of barriers such as many residents having limited technology skills, lack of access to computers, limited staff physically at the building who could provide information support to residents who wanted to participate, and restrictions on in-person meetings that would allow us to provide education about how to use a home blood pressure monitor. We brainstormed ideas for overcoming these barriers with our housing partner. However, they were also experiencing many new demands and they requested that we pause our program until in-person visits could be safely resumed.

\section{Legal considerations}

We continuously monitored COVID-19 cases in our local community as well as regulations by our institution, city, state, and Centers for Disease Control and Prevention.

\section{Community members}

The residents who participate in this program are adults who reside at the low-income housing apartment building. Many residents have multiple chronic health conditions that could place them at higher risk for serious illness from COVID-19. The residents frequently experience challenges engaging with the traditional health care system such as limited transportation, lack of insurance coverage, and distrust of medical professionals. The services that we provide focus on blood pressure and blood glucose screening and education. Our team was not yet prepared or equipped to conduct COVID-19 testing.

\section{Outcome}

We kept in contact with our housing partner on approximately a monthly basis to discuss whether or not it was safe and feasible to resume in-person health events. We agreed to pilot a small, outdoor event in October 2020 as all of the legal requirements were met and stakeholders from both organizations agreed the benefits to residents and students of holding the event outweighed the risks associated with COVID-19. As this was the first event after approximately six months, we limited participating to pharmacy students and the pharmacy and social work preceptors. We determined that the event was successful for residents, students, preceptors and the housing partner staff therefore we proceeded with an additional outdoor event in November 2020 that included pharmacy and social work students and preceptors. We will continue to maintain close communication among all stakeholders to ensure that we carefully weigh the potential benefits and risks each month. 